\title{
The role of calcium and calcium/calmodulin-dependent kinases in skeletal muscle plasticity and mitochondrial biogenesis
}

\author{
Eva R. Chin \\ Department of Cardiovascular and Metabolic Diseases, Pfizer Global Research \& Development, Eastern Point Rd, \\ MS8220-3120, Groton, CT 06340, USA
}

\begin{abstract}
Intracellular $\mathrm{Ca}^{2+}$ plays an important role in skeletal muscle excitation-contraction coupling and also in excitation-transcription coupling. Activity-dependent alterations in muscle gene expression as a result of increased load (i.e. resistance or endurance training) or decreased activity (i.e. immobilization or injury) are tightly linked to the level of muscle excitation. Differential expression of genes in slow- and fast-twitch fibres is also dependent on fibre activation. Both these biological phenomena are, therefore, tightly linked to the amplitude and duration of the $\mathrm{Ca}^{2+}$ transient, a signal decoded downstream by $\mathrm{Ca}^{2+}$-dependent transcriptional pathways. Evidence is mounting that the calcineurin-nuclear factor of activated T-cells pathway and the $\mathrm{Ca}^{2+}$ /calmodulin-dependent kinases (CaMK) II and IV play important roles in regulating oxidative enzyme expression, mitochondrial biogenesis and expression of fibre-type specific myofibrillar proteins. CaMKII is known to decode frequency-dependent information and is activated during hypertrophic growth and endurance adaptations. Thus, it was hypothesized that CaMKII, and possibly CaMKIV, are down regulated during muscle atrophy and levels of expression of CaMKII $\alpha$, -II $\beta$, -II $\gamma$ and -IV were assessed in skeletal muscles from young, aged and denervated rats. The results indicate that CaMKII $\gamma$, but not CaMKII $\alpha$ or $-\beta$, is up regulated in aged and denervated soleus muscle and that CaMKIV is absent in skeletal but not cardiac muscle. Whether CaMKII $\gamma$ up-regulation is part of the pathology of wasting or a result of some adaptational response to atrophy is not known. Future studies will be important in determining whether insights from the adaptational response of muscle to increased loads will provide pharmacological approaches for increasing muscle strength or endurance to counter muscle wasting.
\end{abstract}

Calcium ions: $\mathrm{Ca}^{2+} /$ calmodulin-dependent kinase: Skeletal muscle: Mitochondrial biogenesis

Intracellular $\mathrm{Ca}^{2+}$ plays an important role in signal transduction in all cell types. In skeletal muscle it is well established that $\mathrm{Ca}^{2+}$ plays an essential role in the contraction-relaxation cycle. More recently, the role of $\mathrm{Ca}^{2+}$ in regulating activity-dependent muscle gene expression has been recognized as being important in muscle plasticity and in explaining muscle fibre type heterogeneity (Berchtold et al. 2000; Olson \& Williams, 2000). Neural activation of skeletal muscle results in the release of acetylcholine from the neuro-muscular junction and depolarization of the plasma membrane, which activates force production by a process known as excitation-contraction coupling (see Fig. 1). The frequency and duration of stimulation determine the amplitude and duration of the $\mathrm{Ca}^{2+}$ transients and, as a result, the level of force output by the muscle (Westerblad \& Allen, 1991). Similarly, it is thought that the amplitude and duration of the $\mathrm{Ca}^{2+}$ transient will also determine the set of genes expressed, thus providing a mechanism for tightly coupling the extent of muscle excitation to regulation of transcription (i.e. excitation-transcription coupling). It is this mechanism that may tightly link muscle gene expression to the activation history of the cell, thus providing a basis by which muscles can adapt, at the molecular level, to the functional demands placed on them.

\section{Role of calcium signalling in excitation-contraction coupling and excitation-transcription coupling}

It is well established that the preceding pattern of muscle excitation plays an important role in determining the

\footnotetext{
Abbreviations: $\left[\mathrm{Ca}^{2+}\right]_{\mathrm{i}}$, intracellular free $\mathrm{Ca}^{2+}$ concentrations; $\mathrm{CaMK}, \mathrm{Ca}^{2+} /$ calmodulin-dependent kinase; CnA, calcineurin; $\mathrm{NFAT}$, nuclear factor of activated T-cells; PGC-1, PPAR $\gamma$ co-activator-1; PKC, protein kinase C; SOL, soleus.

Corresponding author: Dr Eva R. Chin, fax +18607154706 , email eva_r_chin@groton.pfizer.com
} 


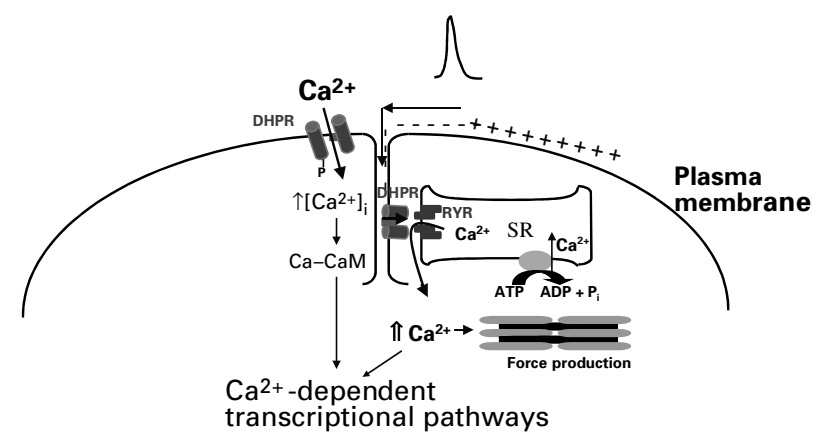

Fig. 1. Skeletal muscle excitation-contraction and excitationtranscription coupling. Activation of muscle contraction results in depolarization of the plasma membrane and the transverse tubule (t-tubule) system. The t-tubule system carries the signal to the interior of the fibre where the voltage-sensing dihydropyridine receptor (DHPR) detects the change in membrane potential and transmits the signal to the calcium-release channel, also known as the ryanodine receptor (RYR). Release of calcium from the internal storage site (sarcoplasmic reticulum; SR) into the myoplasm results in actin-myosin interaction, fibre shortening and force production. Skeletal muscle calcium levels are also elevated by ligand-mediated activation of L-type calcium channels by signalling molecules such as insulin-like growth factor-I, which result in influx of calcium via the plasma membrane. Both sources of intracellular calcium are thought to play a role in excitation-transcription coupling. $\left[\mathrm{Ca}^{2+}\right]_{\mathrm{i}}$, intracellular free calcium ion concentrations; CaM, calmodulin.

pattern of genes expressed, and ultimately in the contractile behaviour of a given muscle (see Pette \& Staron, 1997). The classic cross-innervation experiments of Vrbova (1963) and models using neural or direct muscle stimulation to transform fast skeletal muscle to a slow type demonstrated that muscle fibres can alter their expression of contractile, metabolic and membrane-bound signalling proteins in response to altered input (for review, see Pette \& Vrbova, 1992). Physical activity, through increased loading (i.e. resistance training), increased repetitive stimulation (i.e. endurance training) or decreased physical activity as a result of disuse atrophy, injury or age-related muscle wasting can also alter skeletal muscle phenotype, which in turn is directly related to changes in the proteins expressed in individual fibres. While the ability to alter muscle fibre type characteristics has been well established over the past few decades, the molecular mechanism(s) underlying these adaptive responses are not as well understood. Likewise, the broad heterogeneity between skeletal muscle fibre types is well established. Muscles consist of two main fibre types, slow (type I or slow oxidative) and fast (type II or glycolytic with a varying range of oxidative potential), which vary in their contractile speed, metabolic profile and fatigue resistance. The main fibre types are determined by the myosin heavy chain isoform expressed (types I, IIa, IIx and IIb) and also by alterations in various myofibrillar proteins, including myosin light chains, troponin subunits $\mathrm{I}, \mathrm{T}$ and $\mathrm{C}$ and tropomyosin. While the complement of fibre type-specific genes expressed in a slow fibre $v$. a fast fibre programme is well understood (see Schiaffino \& Reggiani, 1996), the regulatory signals that control differential gene expression between fibre types are less established. It has been hypothesized that the pattern of genes expressed in a fibre type-specific fashion and the changes in gene expression with altered activity or load may be associated with the level of electrical activation, alterations in intracellular metabolites (i.e. glucose, glycogen, ATP), ionic species $\left(\mathrm{H}^{+}, \mathrm{K}^{+}, \mathrm{Ca}^{2+}\right)$ and reactive oxygen species or increased secretion of autocrine and paracrine factors (i.e. insulinlike growth factor-I). Currently, there is great interest in the role of intracellular $\mathrm{Ca}^{2+}$ and $\mathrm{Ca}^{2+}$-dependent regulation of skeletal muscle gene expression.

\section{Role of intracellular calcium ions in regulating skeletal muscle gene expression}

Increased electrical activity of muscle results in a marked elevation of intracellular $\mathrm{Ca}^{2+}$ and this increase is thought to be the primary regulator of altered gene expression in skeletal muscle. Under resting conditions, intracellular free $\mathrm{Ca}^{2+}$ concentrations $\left(\left[\mathrm{Ca}^{2+}\right]_{\mathrm{i}}\right)$ measured in isolated single muscle fibres are 30-50 nM (Westerblad \& Allen, 1991). In contrast, when muscles are activated to contract, $\left[\mathrm{Ca}^{2+}\right]_{\mathrm{i}}$ reaches 100-300 nM (Chin \& Allen, 1996) in slow-twitch (type I) fibres activated at frequencies of slow motor units (10-30 Hz; Hennig \& Lomo 1985), and even higher to 1-2 $\mu_{\mathrm{M}}$ (Westerblad \& Allen, 1991) in fast-twitch (types IIb and IIa) fibres activated at frequencies of fast motor units (80-150 Hz; Hennig \& Lomo, 1985). These dramatic increases in amplitude, as well as the duration for which these amplitudes are achieved, are thought to encode a signal that will be recognized by different downstream $\mathrm{Ca}^{2+}$. dependent transcriptional pathways. The key signalling pathways downstream of the elevation in intracellular $\mathrm{Ca}^{2+}$ that translate this signal into a transcriptional response include the $\mathrm{Ca}^{2+}$-dependent phosphatase calcineurin $(\mathrm{CnA}$; Chin et al. 1998; Naya et al. 2000; Wu et al. 2001), $\mathrm{Ca}^{2+}$ / calmodulin-dependent kinases (CaMK) II (Flück et al. 2000) and IV (Wu et al. 2002) and $\mathrm{Ca}^{2+}$-dependent protein kinase C (PKC; Freyssenet et al. 1999; see Fig. 2). The downstream target genes that are activated by these pathways are many. $\mathrm{Ca}^{2+}$-dependent changes in muscle gene expression have been demonstrated for the nicotinic acetylcholine receptor (Walke et al. 1994), GLUT4 (Ojuka et al. 2002b), sarcoplasmic reticulum $\mathrm{Ca}^{2+}$-transporting ATPase, myosin heavy chain isoforms (Allen et al. 2001; Allen \& Leinwand, 2002) and oxidative enzymes, as well as genes that regulate mitochondrial biogenesis (Freyssenet et al. 1999; Ojuka et al. 2002a, 2003; Chin et al. 2003). These genes are up-regulated (GLUT4; myosin heavy chain types IIa $>>\mathrm{IId} / \mathrm{x}>\mathrm{IIb}$; mitochondrial enzymes cytochrome $c$, cyclooxygenase 1, 5-aminolevulinate synthase, succinate dehydrogenase) or down-regulated (nicotinic acetylcholine receptor, sarcoplasmic reticulum $\mathrm{Ca}^{2+}$-transporting ATPase) with increased contractile activity. These genes also have varied levels of expression between fibres that have high fatigue resistance (types I and IIa) and those of low fatigue resistance (type IIb), supporting the notion that the extent of muscle activation, through the resultant amplitude and duration of $\mathrm{Ca}^{2+}$ elevation, determines the pattern of gene expression in muscle. 


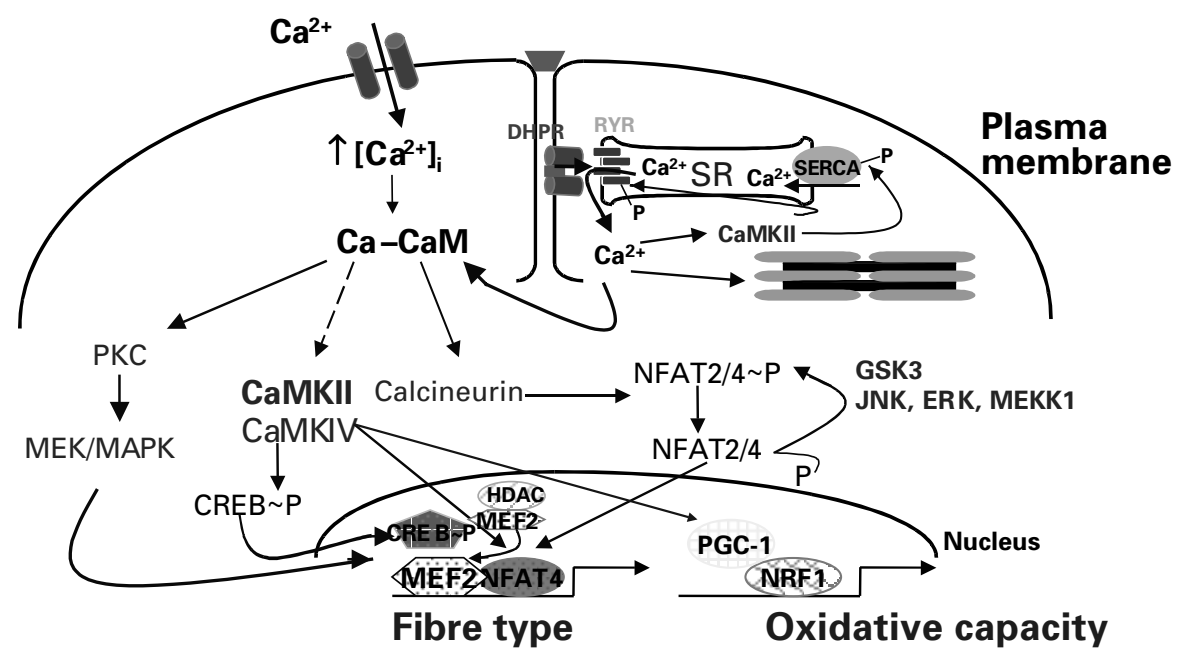

Fig. 2. Calcium-dependent transcriptional pathways in skeletal muscle. Elevations in intracellular calcium result in activation of second messenger signalling pathways, post-transcriptional modification of transcription factors, binding of transcription factors to respective cis-elements and activation of downstream target genes. In skeletal muscle, the primary calcium-dependent transcriptional pathways are the calcineurin-nuclear factor of activated T-cells-(NFAT), calcium/calmodulin-dependent kinases (CaMK) II and IV and the protein kinase C (PKC) pathways. These pathways activate transcription factors that regulate expression of mitochondrial and nuclear-encoded genes with resultant adaptations of both muscle fibre type and oxidative capacity. $\left[\mathrm{Ca}^{2+}\right]$, intracellular free calcium ion concentrations; $\mathrm{CaM}$, calmodulin; CREB, CAMP-response element-binding protein; DHPR, dihydropyridine receptor; ERK, extracellular signal-regulated kinase; GSK3, glycogen synthase kinase 3; HDAC, histone deacetylase; MEF2, myocyte-enhancing factor; MAPK, mitogen-activated protein kinase; MEK, MAPK/ERK kinase; MEKK1, MEK kinase 1; JNK, c-jun N-terminal kinase; NRF, nuclear respiratory factor; $\sim P$, high-energy phosphate; PCG1, PPAR $\gamma$ co-activator-1; RYR, ryanodine receptor; SERCA, sarcoplasmic reticulum calcium ATPase; SR, sarcoplasmic reticulum).

Support for the $\mathrm{Ca}^{2+}$ dependence of mitochondrial enzyme expression has primarily come from studies using cultured myocytes in which $\left[\mathrm{Ca}^{2+}\right]_{\mathrm{i}}$ is elevated by $\mathrm{Ca}^{2+}$-mobilizing agents (i.e. $\mathrm{Ca}^{2+}$ ionophores, caffeine; Freyssenet et al. 1999; Ojuka et al. 2002a, 2003). These studies show increased expression of mitochondrial enzymes at the mRNA and protein level, implicating a transcriptional response to elevated $\mathrm{Ca}^{2+}$. This transcriptional response has been verified using promoter reporter constructs (Freyssenet et al. 1999) that are activated in response to elevated $\left[\mathrm{Ca}^{2+}\right]_{\mathrm{i}}$. Increased expression of transcription factors known to play a role in mitochondrial biogenesis, PPAR $\gamma$ co-activator-1 (PGC-1), mitochondrial transcription factor $\mathrm{A}$, and nuclear respiratory factors 1 and 2 (Ojuka et al. 2003), further supports the role of $\mathrm{Ca}^{2+}$ in regulating mitochondrial gene expression at the transcriptional level. In vivo studies with transgenic mice that overexpress or have disrupted expression of the $\mathrm{Ca}^{2+}$-buffering protein parvalbumin further support the $\mathrm{Ca}^{2+}$ dependence of mitochondrial enzyme expression. Mice with increased expression of parvalbumin, and consequently attenuated levels of $\left[\mathrm{Ca}^{2+}\right]_{\mathrm{i}}$ in activated muscles, have decreased activity of succinate dehydrogenase, a mitochondrial marker enzyme (Chin et al. 2003). These data are consistent with the increased mitochondrial volume and cytochrome $c$ oxidase activity observed in parvalbumin-deficient mice
(Chen et al. 2001). In both the parvalbumin-overexpression and parvalbumin-deficient mice alterations in muscle oxidative potential were independent of changes in myosin isoform, suggesting differential regulation of mitochondrial and contractile genes when $\left[\mathrm{Ca}^{2+}\right]_{\mathrm{i}}$ is regulated physiologically through altered $\mathrm{Ca}^{2+}$-regulatory proteins.

\section{Role of the calcineurin-nuclear factor of activated T-cells pathway in skeletal muscle gene expression}

It has been hypothesized that the $\mathrm{CnA}$-nuclear factor of activated T-cells (NFAT) pathway plays an important role in decoding the $\mathrm{Ca}^{2+}$ amplitude and duration signal to regulate genes differentially expressed in slow fibres $v$. fast fibres (Chin et al. 1998; Naya et al. 2000). This pathway has also been shown to play an important role in the hypertrophic growth response in skeletal muscle (Dunn et al. 1999) as well as in cardiac muscle (Molkentin et al. 1998). The CnA-NFAT pathway was first shown to be a decoder of the amplitude and duration of the $\mathrm{Ca}^{2+}$ signal in T lymphocytes (Dolmetsch et al. 1997) in which it activates the $I L-2$ gene under conditions of a lowamplitude long-duration $\mathrm{Ca}^{2+}$ signal. Under these conditions CnA dephosphorylates the transcription factor NFAT, which unmasks a nuclear localization signal and allows NFAT entry into the nucleus where it can bind to a 
transcriptional complex that activates IL-2 expression. The CnA-NFAT pathway is not, however, activated when $\mathrm{Ca}^{2+}$ pulses reach high-amplitude peaks for short durations and thus does not stimulate IL-2 expression under these conditions. It has now been shown that the CnA-NFAT pathway is activated in single skeletal muscle fibres in a manner also dependent on the level of $\mathrm{Ca}^{2+}$ elevation (Liu et al. 2001). Liu et al. (2001) have shown that CnA-NFAT activation, as determined by NFAT nuclear localization, occurs in single adult muscle fibres in response to continuous stimulation at $10 \mathrm{~Hz}$ or to $5 \mathrm{~s}$ trains of $10 \mathrm{~Hz}$ stimuli every $50 \mathrm{~s}$, a pattern representative of slow fibre recruitment. However, the CnA-NFAT pathway is not activated in response to continuous stimulation at $1 \mathrm{~Hz}$ or with trains of $50 \mathrm{~Hz}$ stimuli every $50 \mathrm{~s}$, the latter of which is representative of a fast-fibre-type recruitment pattern. The nuclear localization of NFAT is inhibited by the CnA inhibitor cyclosporin, implicating the CnA-NFAT pathway.

There is mounting evidence in skeletal muscle that $\mathrm{Ca}^{2+}$ signals through $\mathrm{CnA}$ and downstream through the transcription factors NFAT and myocyte-enhancing factor 2 . In cultured myotubes in vitro it has been shown that slowfibre-specific promoters (i.e. troponin I slow, myoglobin) containing NFAT-binding elements as well as myocyteenhancing factor 2-binding sites are differentially activated compared with fast-fibre-specific promoters that do not have NFAT elements (Chin et al. 1998). Other investigators have suggested that NFAT and myocyte-enhancing factor 2 sites alone cannot account for the preferential activation of the myosin heavy chain isoform expressed in fast oxidative (type IIa) fibres and suggest that additional ciselements and putative transcription factors exist downstream of the $\mathrm{Ca}^{2+}-\mathrm{CnA}$ signal (Allen et al. 2001). Studies in transgenic mice that overexpress an activated form of $\mathrm{CnA}\left(\mathrm{CnA}^{*}\right)$ in skeletal muscle further support the differential activation of slow and oxidative genes (Naya et al. 2000; Wu et al. 2001). In these mice, muscles that express the transgene have higher levels of slow-twitch fibres and express proportionately higher levels of the slow oxidative fibre markers myoglobin, troponin I slow and sarcomeric mitochondrial creatine kinase; in contrast, they express relatively lower levels of the fast glycolytic fibre markers PV and muscle creatine kinase (Naya et al. 2000). Furthermore, this response has been shown to be dosedependent (Wu et al. 2001). Utrophin, an important component of the dystro-sarcoglycan complex that is important for maintaining sarcolemmal membrane integrity, is more highly expressed in slow oxidative fibres and this expression has recently been shown to be CnA-NFAT dependent (Chakkalakal et al. 2003). Utrophin expression is up regulated in $\mathrm{CnA}^{*}$ transgenic mice in the absence of changes in myosin heavy chain, indicating that this gene is regulated by $\mathrm{CnA}-\mathrm{NFAT}$ signalling independent of muscle fibre type. In vivo studies with fast and slow muscle promoter constructs, however, do not support this differential activation of slow-fibre genes in response to activated CnA (Swoap et al. 2000), and suggest that under endogenous regulation $\mathrm{CnA}$ is probably not the only regulator of fibre-type-specific gene expression.

\section{Role of calcium/calmodulin-dependent kinases in regulating skeletal muscle gene expression}

Other pathways shown to play an important role in the $\mathrm{Ca}^{2+}$-dependent regulation of muscle gene expression are the CaMK pathways. The CaMKII pathway has been shown to play an important role in activation of mitochondrial biogenesis and the regulation of oxidative enzyme expression as well as a muscle hypertrophy response (Flück et al. 2000). The CaMKIV pathway has also been shown to play a role in mitochondrial biogenesis (Wu et al. 2002). Examination of $\mathrm{Ca}^{2+}$-dependent and $\mathrm{Ca}^{2+}$-independent CaMKII activity in muscles following $7 \mathrm{~d}$ of stretch overload and following 2 weeks of voluntary wheel running (Flück et al. 2000) has shown that stretch overload of chicken anterior latissimus dorsi muscle results in a $122 \%$ increase in muscle protein content and is associated with a $47 \%$ increase in $\mathrm{Ca}^{2+}$-independent CaMKII activity. $\mathrm{Ca}^{2+}$-dependent and total CaMKII activities are not increased, indicating that there is greater activation of the pre-existing CaMKII protein. Similarly, voluntary wheel running by Sprague-Dawley rats results in a $43 \%$ increase in $\mathrm{Ca}^{2+}$-independent CaMKII activity with no increase in total CaMKII activity. CaMKII has been shown to phosphorylate serum response factor, which can bind to the serum response element and thus activate actin gene expression. The similarity in the response of CaMKII to endurance training and stretch overload suggests that this pathway is upstream of the specific adaptations to these contractile demands. Nonetheless, they do reflect an increase in CaMKII autonomous activity, indicating sustained activation of this $\mathrm{Ca}^{2+}$ signalling pathway.

CaMKII has been shown in other cell types, primarily neurons, to play an important role in decoding the frequency of $\mathrm{Ca}^{2+}$ oscillations in transcriptional regulation (see Braun \& Schulman, 1995; Soderling et al. 2001). Synaptic plasticity, such as long-term potentiation, is triggered by increases in intracellular $\mathrm{Ca}^{2+}$ and activation of CaMKII. The structural organization and molecular regulation of CaMKII allows the enzyme to decode the frequency of $\mathrm{Ca}^{2+}$ spikes into distinct amounts of kinase activity and subsequently into graded levels of transcriptional activation. CaMKII is a multimeric enzyme composed of twelve subunits arranged in two sets of six subunits in a spoke and wheel pattern (see Braun \& Schulman, 1995; Soderling et al. 2001). The central hub portion contains the carboxy terminus association domain and the spoke portions contain the amino terminus catalytic domain and the intervening regulatory domains. When $\left[\mathrm{Ca}^{2+}\right]_{\mathrm{i}}$ is elevated, $\mathrm{Ca}^{2+}$ associates with calmodulin, the $\mathrm{Ca}^{2+}$-calmodulin complex binds to the calmodulinbinding domain and activates intramolecular autophosphorylation, which results in autonomous (i.e. $\mathrm{Ca}^{2+}$ independent) activity. Both the multimeric structure and its regulation by autophosphorylation allow this signalling molecule to titrate levels of kinase activity and therefore transcriptional activation in proportion to $\mathrm{Ca}^{2+}$ oscillatory frequency, amplitude and duration. As a result of its functional role in neurons in decoding frequency-dependent information (De Koninck \& Schulman, 1998) and its observed autophosphorylation following prolonged skeletal 
muscle activation (Fluck et al. 2000), CaMKII is postulated to be the stimulation-frequency decoder in skeletal muscle. Recent evidence also implicates CaMKIV in the $\mathrm{Ca}^{2+}$ dependent regulation of muscle gene expression, specifically in the activation of mitochondrial biogenesis and oxidative enzyme expression (Wu et al. 2002). Overexpression of a constitutively-active form of CaMKIV in mouse skeletal muscle results in increased mitochondrial volume, increased expression of both mitochondrial and nuclear DNA-encoded mitochondrial enzymes involved in fatty acid metabolism and electron transport, and enhanced recovery from fatigue ( $\mathrm{Wu}$ et al. 2002). The mice also show a subtle (approximately $8 \%$ ) increase in slow-twitch fibres. Although the downstream target(s) of CaMKIV has not been identified, it is known that cAMP-response element-binding protein is phosphorylated in CaMKIVmediated transcription in neurons and T-cells (Matthews et al. 1994). The role of cAMP-response element-binding protein phosphorylation by CaMKIV in regulating skeletal muscle gene expression has not been examined. In CaMKIV transgenic mice the increase in mitochondrial content is, however, associated with increased expression of PGC-1, the master switch of mitochondrial biogenesis. Transgenic mice that overexpress PGC-1 in skeletal muscle also show a similar phenotype, with increased oxidative fibre content and increased expression of slow type I fibre genes (Lin et al. 2002). Thus, CaMKIV appears to signal through PGC-1 to increase mitochondrial biogenesis in skeletal muscle. Interestingly, muscle energy depletion using a $\beta$-guanidinopropionic acid diet to deplete creatine phosphate stores results in increased PGC-1 and CaMKIV expression concomitant with mitochondrial biogenesis (Zong et al. 2002). Under these conditions, up-regulation of CaMKIV and PGC-1 is dependent on AMP kinase activity, since AMP kinase-dominant negative mice do not show these mitochondrial adaptations with $\beta$-guanidinopropionic acid treatment (Zong et al. 2002). It is likely that both the decrease in cellular ATP and the increase in intracellular $\mathrm{Ca}^{2+}$ are responsible for signalling an increase in mitochondrial biogenesis and muscle oxidative capacity in response to repetitive muscle activation.

\section{Calcium/calmodulin-dependent kinases as therapeutic drug targets}

Based on the evidence implicating CaMK signalling in muscle fibre type determination, muscle oxidative capacity and muscle adaptation to overload hypertrophy and endurance activity, there is much interest in the potential role of CaMK modulators as a pharmacological approach for muscle myopathies. Conditions of muscle atrophy, including disuse atrophy, injury and age-related muscle wasting, may be attenuated with agonists of a target molecule such as CaMKII or CaMKIV that could increase strength or oxidative capacity. While CaMKIV appears to be a good candidate for pharmacological intervention, based on the biological response in mice to CaMKIV overexpression (Wu et al. 2002), its expression level in muscle is not well documented. CaMKII may also be a good target, but it is not clear which of its multiple isoforms is most important in skeletal muscle signalling. CaMK II isoforms arise from four different genes that are differentially expressed, with CaMKII $\alpha$ and CaMKII $\beta$ expressed in neural tissue (Braun \& Schulman, 1995) and muscle (Bayer et al. 1998), and CaMKII $\gamma$ and CaMKII $\delta$ expressed ubiquitously (Braun \& Schulman, 1995). CaMKI has not been studied extensively in skeletal muscle, although it has been shown that activated CaMKI is equally as effective as CaMKIV in activating a myoglobin promoter whereas CaMKII does not transcriptionally activate myoglobin (Wu et al. 2002). Table 1 summarizes the major CaMK enzymes and their regulatory properties. Based on their biochemical properties and their potential biological role in skeletal muscle adaptation, the expression patterns of CaMKII and CaMKIV in skeletal muscle have been further examined; in particular, to determine whether CaMKII or CaMKIV expression levels are altered in age-related muscle wasting or in denervation atrophy.

In skeletal muscle samples obtained from soleus (SOL), extensor digitorum longus or tibialis anterior muscles of young or aged (4 and 27 months respectively) Harlan Sprague-Dawley rats and from young rats after $20 \mathrm{~d}$ denervation, it has been found that by 27 months of age these rats show a $33 \%$ decrease in muscle mass and maximum tetanic force (C Ibebunjo, unpublished results). Analysis of CaMKII and CaMKIV mRNA levels (by RT-PCR using primers specific for the various CaMKII isoforms and for CaMKIV; see Fig. 3 legend) and CaMKII protein levels (by Western blot using antibodies obtained from Santa Cruz, Santa Cruz, CA, USA) has shown that CaMKII $\alpha$ and CaMKII $\beta$ mRNA levels are not altered in SOL and extensor digitorum longus muscle from aged rats compared with young rats (Fig. 3), but interestingly there are two CaMKII $\beta$ splice variants, one of which is expressed at lower levels in the fast-twitch extensor digitorum longus compared with the slow-twitch SOL. CaMKIV mRNA levels are not detectable in SOL or extensor digitorum longus under conditions (thirty cycles) in which mRNA

Table 1. Expression pattern and biochemical properties of the calcium ion/calmodulin(CaM)-dependent kinases (CaMk)

\begin{tabular}{|c|c|c|c|}
\hline & CaMKI & CaMKII & CaMKIV \\
\hline Tissue distribution & Ubiquitous & Ubiquitous & Limited \\
\hline Localization & Cytoplasmic & $\begin{array}{l}\text { Cytoplasmic } \\
\quad(\text { except } \alpha \mathrm{B}, \gamma \mathrm{A} \delta \mathrm{B})\end{array}$ & $\begin{array}{l}\text { Cytoplasmic and } \\
\text { nuclear }\end{array}$ \\
\hline Subunit composition & Monomeric & Homo- or hetero-multimeric & Monomeric \\
\hline $\mathrm{Ca}^{2+}$ independence & No & Yes (to $80 \%$ ) & Yes (to $20 \%$ ) \\
\hline \multicolumn{4}{|l|}{$K_{\mathrm{CaM}}$} \\
\hline Unphosphorylated & $14 \mathrm{~nm}$ & $20-100 \mathrm{~nm}$ & $158 \mathrm{~nm}$ \\
\hline Phosphorylated & $4 \mathrm{~nm}$ & $60 \mathrm{pm}$ & \\
\hline
\end{tabular}



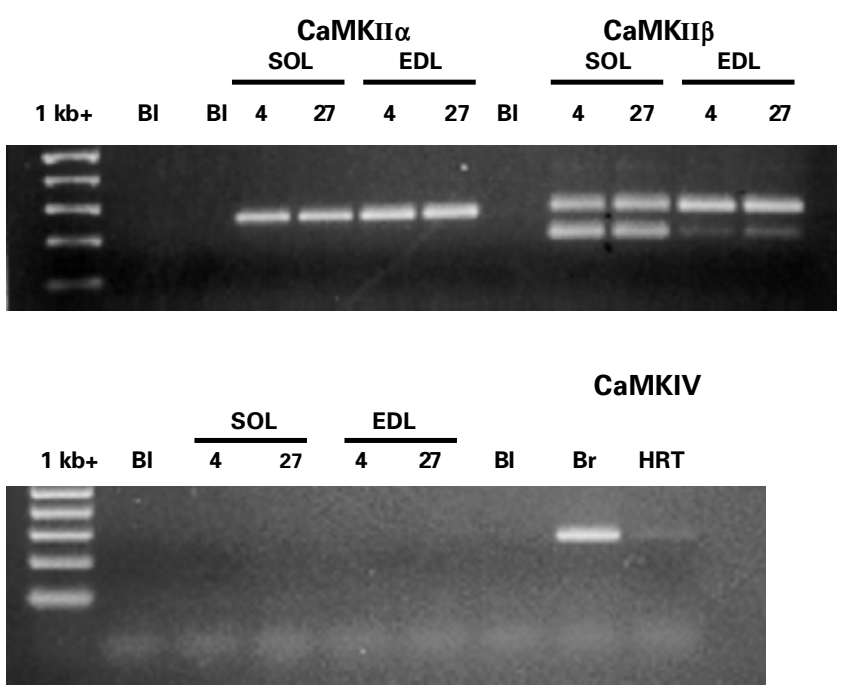

Fig. 3. Calcium/calmodulin-dependent kinases (CaMK) II and IV mRNA expression in young and aged rat skeletal muscle. Soleus $(\mathrm{SOL})$ and extensor digitorum longus (EDL) muscles were obtained from young (4 months) and aged (27 months) Sprage-Dawley rats. Total RNA was isolated using TriPure reagent (Roche, Basel, Switzerland) and $10 \mathrm{ng}$ used to generate cDNA with Mulroney murine leukaemic virus RT in a $60 \mu$ l reaction. cDNA $(5 \mu l)$ was amplified with Taq polymerase and the following CaMK primers: GAATGACAGCCTTTGAACCGG and TGCCATTTTCCATCCCTGC for CaMKIl $\alpha$ forward and reverse respectively; ATGCAAGGAGGAAGCTCAAGG and TGGATAACGGTGGTTTGAGGC for CaMKII $\beta$ forward and reverse respectively; CAGGAAAAGCAGCCAACTTTG and TAGCTTCATCGTCTCATCGGC for CaMKIV forward and reverse respectively. PCR conditions were $94^{\circ} \mathrm{C}$ for $3 \mathrm{~min} ; 94^{\circ} \mathrm{C}$ for $30 \mathrm{~s}, 60^{\circ} \mathrm{C}$ for $1 \mathrm{~min}, 72^{\circ} \mathrm{C}$ for $1 \mathrm{~min} \times$ thirty cycles; $72^{\circ} \mathrm{C}$ for $3 \mathrm{~min}$. PCR products were run on agarose gels $(8 \mathrm{ml} / \mathrm{l})$ alongside a $1 \mathrm{~kb}+\mathrm{DNA}$ standard. $\mathrm{BI}$, blank; $\mathrm{Br}$, brain; HRT, heart.

is detected in heart and brain. The absence of evidence of CaMKIV expression in skeletal muscle is consistent with previous reports in the literature (see Matthews et al. 1994). A recent report by Zong et al. (2002) is the first to show CaMKIV expression in skeletal muscle, but these data are inconsistent with the observations from the author's laboratory.

Examination of the changes in CaMKII expression at the protein level using commercially-available antibodies has failed to detect either CaMKII $\alpha$ or CaMKII $\beta$; however, CaMKII $\gamma$ has been detected and analysed in young, aged and denervated muscles. Fig. 4 shows that CaMKII $\gamma$ is up regulated approximately 2 -fold in aged and denervated SOL muscle. Since CaMKII is activated during a hypertrophic and endurance training response, it was hypothesized that CaMKII expression would be decreased during periods of muscle atrophy. These findings do not support this hypothesis and suggest that SOL muscle atrophy may activate a compensatory adaptive response to counter the ongoing muscle wasting. Alternatively, CaMKII $\gamma$ protein levels could be increased despite decreased enzymic activity. Fig. 5 illustrates the changes in CaMKII $\gamma$ protein levels in tibialis anterior muscle of aged and denervated animals. In contrast to the SOL, this muscle shows no

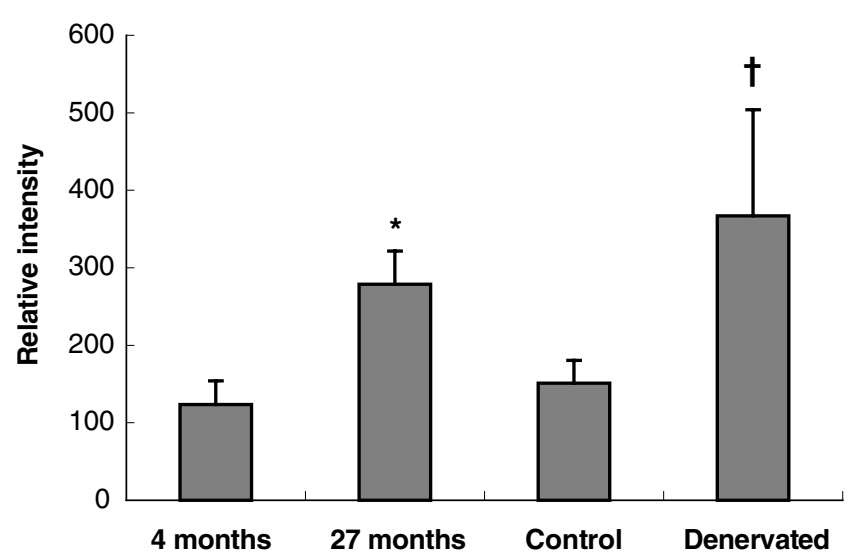

Fig. 4. Calcium/calmodulin-dependent kinases (CaMK) Il $\gamma$ protein expression in young, aged and denervated rat soleus muscle. Soleus muscles were obtained from young (4 months) or aged (27 months) Sprague-Dawley rats and from young rats $20 \mathrm{~d}$ after sciatic nerve transection. Muscles were homogenized in a hypotonic lysis buffer $\left(20 \mathrm{~mm}-\mathrm{Hepes}, 10 \mathrm{~mm}-\mathrm{NaCl}, 1.5 \mathrm{~mm}-\mathrm{MgCl}_{2}, 1 \mathrm{ml}\right.$ Triton X-100/l, $200 \mathrm{ml}$ glycerol/l, $1 \mathrm{~mm}$-dithiothreitol and protease inhibitors complete EDTA-free; Roche, Basel, Switzerland) and $10 \mu \mathrm{g}$ samples were run on $4-12 \%$ Bis-Tris gels in 2-( $N$-morpholino)ethanesulfonic acid-SDS buffer. Protein was transferred onto a $0.45 \mu \mathrm{m}$ nitrocellulose membrane and probed with anti-CaMKIl $\gamma$ antibody (Santa Cruz, Santa Cruz, CA, USA). Western blot images were quantified using a Fujifilm Imager (Fujifilm BAS2500; Fujifilm, Tokyo, Japan). Values are means and standard deviations represented by vertical bars for four samples. Mean value for soleus muscle from aged rats was significantly different from that for soleus muscle from young rats: ${ }^{\star} P<0 \cdot 05$. Mean value for denervated muscle was significantly different from that for the control muscle: $\dagger P<0.05$

change in protein expression with muscle atrophy. Thus, a compensatory adaptation appears to exist only in the slow oxidative muscles. Based on these observations it is not clear whether CaMKII or CaMKIV would be suitable targets for pharmacological intervention for muscle wasting pathologies.

\section{Physiological importance of calcium ion-dependent pathways in skeletal muscle gene expression}

While there is mounting evidence for $\mathrm{Ca}^{2+}$-dependent pathways in regulating activity-dependent changes in gene expression in skeletal muscle, the role played by physiologically-relevant changes in $\left[\mathrm{Ca}^{2+}\right]_{i}$ is not as clear. In studies examining $\mathrm{Ca}^{2+}$-dependent regulation of muscle gene expression, elevations in intracellular $\mathrm{Ca}^{2+}$ are achieved using pharmacological agents in cultured myocytes or the downstream pathways are activated by overexpression of exogenous genes in GM mice. In order to understand the role that these pathways play in vivo, the physiological relevance of these models needs to be considered. Pharmacological tools, primarily $\mathrm{Ca}^{2+}$ ionophores (i.e. A23187 and ionomycin) and caffeine, have been shown to up-regulate slow-fibre-specific and oxidative genes. While this finding does implicate a $\mathrm{Ca}^{2+}$ dependence of the transcriptional response, the cellular 


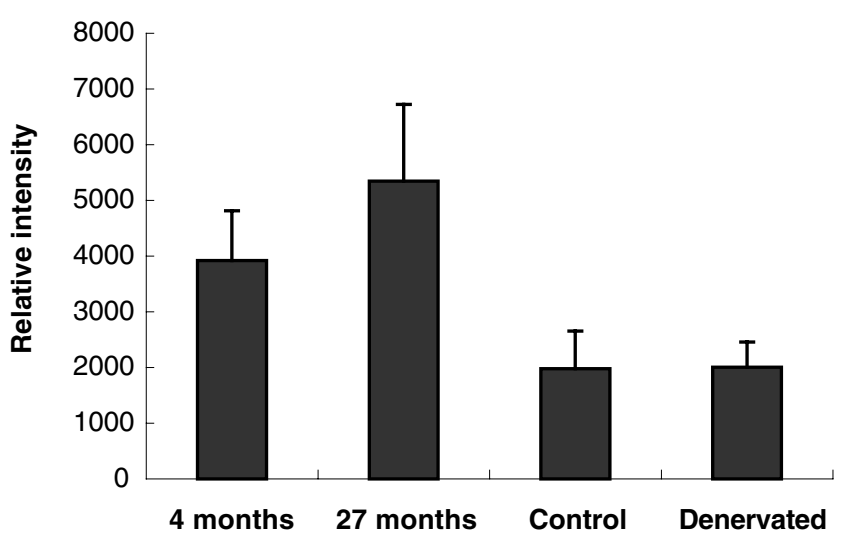

Fig. 5. Calcium/calmodulin-dependent kinases (CaMK) Il $\gamma$ protein expression in young, aged and denervated rat tibialis anterior muscle. Tibialis anterior were obtained from young (4 months) or aged (27 months) Sprague-Dawley rats and from young rats $20 \mathrm{~d}$ after sciatic nerve transection. Muscles were homogenized in a hypotonic lysis buffer (20 mM-Hepes, $10 \mathrm{~mm}-\mathrm{NaCl}, 1.5 \mathrm{~mm}-\mathrm{MgCl}_{2}$, $1 \mathrm{ml}$ Triton X-100/l, $200 \mathrm{ml}$ glycerol//, $1 \mathrm{~mm}$-dithiothreitol and protease inhibitors complete EDTA-free; Roche, Basel, Switzerland) and $10 \mu \mathrm{g}$ samples were run on $4-12 \%$ Bis-Tris gels in 2( $N$-morpholino)ethanesulfonic acid-SDS buffer. Protein was transferred onto a $0.45 \mu \mathrm{m}$ nitrocellulose membrane and probed with anti-CaMKIl $\gamma$ antibody (Santa Cruz, Santa Cruz, CA, USA). Western blot images were quantified using a Fujifilm Imager (Fujifilm BAS2500; Fujifilm, Tokyo, Japan). Values are means and standard deviations represented by vertical bars for four samples. Differences between soleus muscle from aged and young rats and between control and denervated muscle were not statistically significant.

consequences may not reflect those occurring during physiological activation of muscle where the $\mathrm{Ca}^{2+}$ oscillatory pattern is finely regulated. Prolonged exposure to ionophores results in elevation of $\left[\mathrm{Ca}^{2+}\right]_{\mathrm{i}}$ to micromolar levels for prolonged durations, well beyond the physiological range expected during muscle excitation. Ojuka et al. (2002a) have recently demonstrated that continuous exposure $(24 \mathrm{~h} / \mathrm{d})$ for $5 \mathrm{~d}$ to $1 \mu \mathrm{M}$-ionomycin, which elevates $\left[\mathrm{Ca}^{2+}\right]_{\mathrm{i}}$ to $500-600 \mathrm{~nm}$, increases mitochondrial enzyme expression; however, it results in a loss of myocyte protein content and decreased cell viability. Prolonged exposure to caffeine, a ryanodine receptor agonist, also results in decreased growth of myotubes and does not activate slow muscle genes (E Chin, unpublished results). In contrast, intermittent $(5 \mathrm{~h} / \mathrm{d})$ exposure to ionomycin or caffeine increases mitochondrial enzyme expression without decreasing protein content (Ojuka et al. 2002a). Other studies have shown that down-regulation of the nicotinic acetylcholine receptor in response to elevations in $\left[\mathrm{Ca}^{2+}\right]_{\mathrm{i}}$ induced by $\mathrm{A} 23187$ is not suppressed by the CaMKII inhibitor KN93; however, it is suppressed by KN93 when $\left[\mathrm{Ca}^{2+}\right]_{\mathrm{i}}$ is elevated by electrical stimulation (Macpherson et al. 2002). Thus, muscle contractile-induced $\mathrm{Ca}^{2+}$ dependent regulation of gene expression may not always be mimicked by ionophore-induced elevations in $\left[\mathrm{Ca}^{2+}\right]_{i}$ in culture myocytes. Whether this discrepancy reflects the release of $\mathrm{Ca}^{2+}$ from different pools (i.e. from the sarcoplasmic reticulum during contractile activity and from the extracellular pool during ionophore stimulation), or that the finely regulated amplitude and duration of $\mathrm{Ca}^{2+}$ oscillations induced by neural stimulation activate different downstream pathways, is not known. Additionally, muscle contractile activity may invoke $\mathrm{Ca}^{2+}$-independent signals (i.e. mechanical deformation, ATP depletion) that synergize with $\mathrm{Ca}^{2+}$-dependent signals to fully activate contractiledependent adaptive responses.

\section{Summary}

In summary, there is strong evidence that intracellular $\mathrm{Ca}^{2+}$ signals in skeletal muscle are important not only in excitation-contraction coupling but also in excitationtranscription coupling. The specific signalling pathways that are activated in response to differing frequency, amplitude and duration of various $\mathrm{Ca}^{2+}$ transients that signal to different downstream target genes are still not fully understood. Evidence is mounting that the CnA-NFAT pathway as well as CaMK pathways play important roles in activating mitochondrial biogenesis and, to a lesser extent, myosin and other contractile protein isoform expression. It is also clear that multiple signals may converge to fully activate an adaptational response. Transcriptional regulation through $\mathrm{CnA}-\mathrm{NFAT}$ and $\mathrm{CaMK}$ pathways are synergistic (Wu et al. 2001) and $\mathrm{Ca}^{2+}$-dependent $\mathrm{CaMK}$ signals may be synergistic with ATP-dependent signalling through AMP kinase. Through this well-coordinated system of feedforward- and feedback-mediated signalling, skeletal muscles can optimally adapt to the loads placed on them and thus improve functional capacity to meet the demands of the imposed load.

\section{Acknowledgements}

In memory and thanks to Faan Wen Bangerter for technical assistance and support during my early days at Pfizer. You will always be missed. Thanks also to Jim O'Malley for assistance with the denervation studies.

\section{References}

Allen DL \& Leinwand LA (2002) Intracellular calcium and myosin isoform transitions. Calcineurin and calcium-calmodulin kinase pathways regulate preferential activation of the IIa myosin heavy chain promoter. Journal of Biological Chemistry 277, 45323-45330.

Allen DL, Sartorius CA, Sycuro LK \& Leinwand LA (2001) Different pathways regulate expression of the skeletal myosin heavy chain genes. Journal of Biological Chemistry 276, 43524-43533.

Bayer KU, Harbers K \& Schulman H (1998) alphaKAP is an anchoring protein for a novel CaM kinase II isoform in skeletal muscle. EMBO Journal 17, 5598-5605.

Berchtold MW, Brinkmeier H \& Muntener M (2000) Calcium ion in skeletal muscle: its crucial role for muscle function, plasticity, and disease. Physiological Reviews 80, 1215-1265.

Braun AP \& Schulman H (1995) The multifunctional calcium/ calmodulin-dependent protein kinase: from form to function. Annual Review of Physiology 57, 417-445.

Chakkalakal JV, Stocksley MA, Harrison MA, Angus LM, Deschenes-Furry J, St Pierre S, Megeney LA, Chin ER, Michel RN \& Jasmin BJ (2003) Expression of utrophin A mRNA 
correlates with the oxidative capacity of skeletal muscle fiber types and is regulated by calcineurin/NFAT signaling. Proceedings of the National Academy of Sciences USA 100, 7791-7796.

Chen G, Carroll S, Racay P, Dick J, Pette D, Traub I, Vrbova G, Eggli P, Celio M \& Schwaller B (2001) Deficiency in parvalbumin increases fatigue resistance in fast-twitch muscle and upregulates mitochondria. American Journal of Physiology 281, C114-C122.

Chin ER \& Allen DG (1996) The role of elevations in intracellular $\left[\mathrm{Ca}^{2+}\right]$ in the development of low frequency fatigue in mouse single muscle fibres. Journal of Physiology (London) 491, 813-824.

Chin ER, Grange RW, Viau F, Simard AR, Humphries C, Shelton J, Bassel-Duby R, Williams RS \& Michel RN (2003) Alterations in slow-twitch muscle phenotype in transgenic mice overexpressing the $\mathrm{Ca}^{2+}$ buffering protein parvalbumin. Journal of Physiology (London) 547, 649-663.

Chin ER, Olson EN, Yang Q, Shelton J, Bassel-Duby R \& Williams RS (1998) A calcineurin-dependent pathway controls skeletal muscle fiber type. Genes and Development 12, 2499-2509.

De Koninck P \& Schulman H (1998) Sensitivity of CaM kinase II to the frequency of $\mathrm{Ca}^{2+}$ oscillations. Science 279, 227-230.

Dolmetsch RE, Lewis RS, Goodnow CC \& Healy JI (1997) Differential activation of transcription factors induced by $\mathrm{Ca}^{2+}$ response amplitude and duration. Nature 386, 855-858.

Dunn SE, Burns JL \& Michel RN (1999) Calcineurin is required for skeletal muscle hypertrophy. Journal of Biological Chemistry 274, 21908-21912.

Flück M, Waxham MN, Hamilton MT \& Booth FW (2000) Skeletal muscle $\mathrm{Ca}^{2+}$ )-independent kinase activity increases during either hypertrophy or running. Journal of Applied Physiology 88, 352-358.

Freyssenet D, Di Carlo M \& Hood DA (1999) Calciumdependent regulation of cytochrome $\mathrm{c}$ gene expression in skeletal muscle cells. Identification of a protein kinase c-dependent pathway. Journal of Biological Chemistry 274, 9305-9311.

Hennig R \& Lomo T (1985) Firing patterns of motor units in normal rats. Nature 314, 164-166.

Lin J, Wu H, Tarr PT, Zhang CY, Wu Z, Boss O, Michael LF, Puigserver P, Isotani E, Olson EN, Lowell BB, Bassel-Duby R \& Spiegelman BM (2002) Transcriptional co-activator PGC-1 alpha drives the formation of slow-twitch muscle fibres. Nature 418, 797-801.

Liu Y, Cseresnyes Z, Randall WR \& Schneider MF (2001) Activity-dependent nuclear translocation and intranuclear distribution of NFATc in adult skeletal muscle fibers. Journal of Cell Biology 155, 27-39.

Macpherson P, Kostrominova T, Tang H \& Goldman D (2002) Protein kinase $\mathrm{C}$ and calcium/calmodulin-activated protein kinase II (CaMK II) suppress nicotinic acetylcholine receptor gene expression in mammalian muscle. A specific role for CaMK II in activity-dependent gene expression. Journal of Biological Chemistry 277, 15638-15646.

Matthews RP, Guthrie CR, Wailes LM, Zhao X, Means AR \& McKnight GS (1994) Calcium/calmodulin-dependent protein kinase types II and IV differentially regulate CREB-dependent gene expression. Molecular and Cellular Biology 14, $6107-6116$.
Molkentin JD, Lu JR, Antos CL, Markham B, Richardson J, Robbins J, Grant SR \& Olson EN (1998) A calcineurindependent transcriptional pathway for cardiac hypertrophy. Cell 93, 215-228.

Naya FJ, Mercer B, Shelton J, Richardson JA, Williams RS \& Olson EN (2000) Stimulation of slow skeletal muscle fiber gene expression by calcineurin in vivo. Journal of Biological Chemistry 275, 4545-4548.

Ojuka EO, Jones TE, Han DH, Chen M \& Holloszy JO (2003) Raising $\mathrm{Ca}^{2+}$ in L6 myotubes mimics effects of exercise on mitochondrial biogenesis in muscle. FASEB Journal 17, 675-681.

Ojuka EO, Jones TE, Han DH, Chen M, Wamhoff BR, Sturek M \& Holloszy JO (2002a) Intermittent increases in cytosolic $\mathrm{Ca}^{2+}$ stimulate mitochondrial biogenesis in muscle cells. American Journal of Physiology 283, E1040-E1045.

Ojuka EO, Jones TE, Nolte LA, Chen M, Wamhoff BR, Sturek M \& Holloszy JO (2002b) Regulation of GLUT4 biogenesis in muscle: evidence for involvement of AMPK and $\mathrm{Ca}\left({ }^{2+}\right)$. American Journal of Physiology 282, E1008-E1013.

Olson EN \& Williams RS (2000) Remodeling muscles with calcineurin. Bioessays 22, 510-519.

Pette D \& Staron RS (1997) Mammalian skeletal muscle fiber type transitions. International Review of Cytology 170, 143-223.

Pette D \& Vrbova G (1992) Adaptation of mammalian skeletal muscle fibers to chronic electrical stimulation. Reviews in Physiology, Biochemistry and Pharmacology 120, 115-202.

Schiaffino S \& Reggiani C (1996) Molecular diversity of myofibrillar proteins: Gene regulation and functional significance. Physiological Reviews 76, 371-423.

Soderling TR, Chang B \& Brickey D (2001) Cellular signaling through multifunctional $\mathrm{Ca} 2+/$ calmodulin-dependent protein kinase II. Journal of Biological Chemistry 276, 3719-3722.

Swoap SJ, Hunter RB, Stevenson EJ, Felton HM, Kansagra NV, Lang JM, Esser KA \& Kandarian SC (2000) The calcineurinNFAT pathway and muscle fiber-type gene expression. American Journal of Physiology 279, C915-C924.

Vrbova G (1963) The effects of motoneurone activity on the speed of contraction of striated muscle. Journal of Physiology (London) 169, 513-526.

Walke W, Staple J, Adams L, Gnegy M, Chahine K \& Goldman D (1994) Calcium-dependent regulation of rat and chick muscle nicotinic acetylcholine receptor (nAChR) gene expression. Journal of Biological Chemistry 269, 19447-19456.

Westerblad H \& Allen DG (1991) Changes in myoplasmic calcium concentration during fatigue in single mouse muscle fibres. Journal of General Physiology 98, 615-635.

$\mathrm{Wu} \mathrm{H}$, Kanatous SB, Thurmond FA, Gallardo T, Isotani E, Bassel-Duby R \& Williams RS (2002) Regulation of mitochondrial biogenesis in skeletal muscle by CaMK. Science 296, 349-352.

Wu H, Rothermel B, Kanatous S, Rosenberg P, Naya FJ, Shelton JM, Hutcheson KA, DiMaio JM, Olson EN, Bassel-Duby R \& Williams RS (2001) Activation of MEF2 by muscle activity is mediated through a calcineurin-dependent pathway. EMBO Journal 20, 6414-6423.

Zong H, Ren JM, Young LH, Pypaert M, Mu J, Birnbaum MJ \& Shulman GI (2002) AMP kinase is required for mitochondrial biogenesis in skeletal muscle in response to chronic energy deprivation. Proceedings of the National Academy of Sciences USA 99, 15983-15987. 